\title{
New species of Orasema (Hymenoptera: Eucharitidae) from Central and South America
}

\author{
John M. Heraty \& Austin J. Baker
}

To cite this article: John M. Heraty \& Austin J. Baker (2020) New species of Orasema (Hymenoptera: Eucharitidae) from Central and South America, Journal of Natural History, 54:9-12, 735-754, DOI: 10.1080/00222933.2020.1747651

To link to this article: https://doi.org/10.1080/00222933.2020.1747651

册Published online: 23 Sep 2020.

Submit your article to this journal $₫$

Џll Article views: 5

Q View related articles $\asymp$

View Crossmark data $\nearrow$ 


\title{
New species of Orasema (Hymenoptera: Eucharitidae) from Central and South America
}

\author{
John M. Heraty and Austin J. Baker \\ Department of Entomology, University of California, Riverside, CA, USA
}

\begin{abstract}
Six new species of Orasema are described. Four species are placed into a newly recognised lasallei species group: Orasema lasallei sp. nov. (Costa Rica, Honduras, Mexico, Nicaragua), O. janzeni sp. nov. (Costa Rica), O. peckorum sp. nov. (Brazil) and O. vasquezi sp. nov. (Colombia). One species is placed in the wayqecha group, Orasema masneri sp. nov. (Ecuador). One other species is currently unplaced to species group, $O$. Ionginoi sp. nov. (Costa Rica). Orasema lasallei was taken from a nest of Pheidole vorax (Fabr.) and an undescribed species near $O$. longinoi from a nest of Pheidole dossena Wilson (Myrmicinae). The morphology of the digitate labrum is discussed with respect to other Eucharitidae.
\end{abstract}

\section{ARTICLE HISTORY}

Received 29 August 2019

Accepted 20 March 2020

Published online 23

September 2020

Published in print 23

September 2020

\section{KEYWORDS}

Parasitoid; Orasema; Formicidae; Chalcidoidea; labrum; morphology

\section{Introduction}

The genus Orasema Cameron is a monophyletic group of ant parasitoids confined to the New World (Burks et al. 2018; Baker et al. 2020). Confirmed host associations are all with Myrmicinae (Formicidae), with the ancestral host postulated as the genus Pheidole Westwood (Baker et al. 2020). Orasema is diverse and has been divided into several species groups starting with Heraty $(1994,2000)$, with revisions completed for species in the festiva group (Burks et al. 2015), xanthopus group (Heraty et al. 1993), stramineipes group (Burks et al. 2018) and the simulatrix and wayqecha groups (Herreid and Heraty 2017). The lasallei species group described herein is new, one species is added to the wayqecha group, and one new species cannot be placed in existing species groups. Two of these lineages (lasallei group and 0 . longinoi sp. nov.) are represented in the molecular analyses presented in Baker et al. (2020), with four of the molecular voucher species in that paper receiving names herein.

Five of the species being described have an unusual feature for the subfamily, with at least some of the included specimens of these species having more than 4 marginal digits on the labrum (Figure 3a,b). Eucharitidae almost always have a series of long digits along the ventral margin of the labrum (Darling 1988). Eucharitidae usually either have few (4 or rarely 5) or many (6-16) labral digits (Heraty 1994, 2000, 2002). In rare cases, the digits or even the labrum are absent, which can be associated with a general reduction in the mouthparts (Heraty 2002). Akapalinae, Gollumiellinae, the majority of Oraseminae and Psilocharitini (Eucharitinae) have a 4-digitate labrum (Heraty 1994, 2002). Within 
Eucharitini, a 4-digitate labrum is rare (Pseudometagea Ashmead, Lasiokapala Ashmead, Striostilbula Bouček), with most taxa having 9-16 digits (Heraty 1994). Based on these outgroup comparisons, a 4-digitate labrum is almost certainly plesiomorphic for Oraseminae. Among species of Oraseminae with a primarily 4-digitate labrum, occasional specimens can have 5 or more digits. At the generic level, a labrum with more than 6 digits occurs in Ibitya Burks et al. (7-10; Madagascar) and Matantas Burks et al. (6-8; New Caledonia). Other Old World genera generally have 4 digits, but with some species or specimens within a species having a greater number (e.g. Timioderus peridentatus Heraty with 5 or 8 digits, some species of Orasemorpha Bouček with 7 digits, and Losbanus Ishii with up to 7 digits; but all typically with 4). Orasema almost always has a 4-digitate labrum. However, a greater number of digits is found in the Orasema festiva group (8-10), the wayqecha group (7-10), and Orasema tolteca Mann (4-8). Thus, there is a pattern of both consistency and inconsistency in the number of species or groups that have a labrum with more than 5 digits. We herein document extreme within-species variation in the number of digits found within species in the lasallei group that most commonly have more than 6 labral digits.

Based on the phylogenetic hypothesis for Oraseminae presented in Baker et al. (2020), a labrum with more than 6 digits has evolved numerous times in unrelated lineages and thus does not appear to have phylogenetic significance beyond species group recognition in some cases. This is in contrast to the presence of a multidigitate labrum in Eucharitinae, which based on the phylogenetic hypotheses presented in Heraty (2002) and Murray et al. (2013), would represent a single character state change that supports a clade within the tribe Eucharitini after excluding the 4-digitate genus Pseudometagea (sister group to the remainder) and accepting reversals to a 4-digitate labrum in the derived genera Lasiokapala and Striostilbula. It is unclear what drives the consistency and phylogenetic significance of labral morphology in one group and not the other.

Darling (1988) proposed that the digitate labrum in Eucharitidae and Perilampidae was an adaptation for pollen exclusion while nectar feeding, although recognising that the more derived Eucharitini likely do not nectar feed. There is no direct correlation with body size, although larger species sometimes do tend to have a greater number of digits. Within the lasallei species group, there is a large degree of variation in digit number within species, suggesting a high overall level of plasticity, which may be indicative of the changes seen across Oraseminae.

\section{Material and methods}

Material was examined from the UMNH: Utah Museum of Natural History, Salt Lake City, UT, USA (formerly AEIC collection); CNC: Canadian National Collection of Insects, Arachnids \& Nematodes, Ottawa, Canada; MUCR: Universidad de Costa Rica, Museo de Insectos, San Jose, Costa Rica; MHNG: Muséum d'Histoire Naturelle, Geneva, Switzerland; MZLU: Lund University, Lund, Sweden; MEM: Mississippi Entomological Museum, Mississippi State University, Starkville, MS, USA; INBIO: Instituto Nacional de Biodiversidad, Santo Domingo de Heredia, Costa Rica; SEMC: Snow Entomological Museum, University of Kansas, Lawrence, KA, USA; TAMU: Texas A\&M University, College Station, TX, USA. UCRC: UCR Entomology Research Museum, Riverside, CA, USA; USNM: National Museum of Natural History, Washington, DC, USA. 
All specimens are labelled with a UCRCENT barcode label indicating the museum of deposition and a unique specimen identification number. Georeferenced points for specimens estimated from Google Earth are italicised. Photographs were taken using a Leica Imaging System with a Z16 APO A microscope, and stacked using Zerene Stacker (version 1.04, Zerene Systems, LLC). Morphological terms follow Heraty et al. (2018) and Burks et al. (2018). Surface sculptural terms follow illustrations provided by Harris (1979).

\section{Orasema lasallei species group}

\section{Diagnosis}

Recognised from all other species of Orasema by having a combination of 8 funiculars in males and females (Figure 2), the labrum with 4-12 digits (usually $>6$ ), mesothorax dorsally scabrous or coarse rugose, fore wing with a setose costal cell, speculum bare, and basal third of hind wing with scattered microsetae, otherwise pilose. Other features include the yellow scape, yellowish-brown mandibles with a darker brown margin, propodeal callus with 2-3 minute setae, wings hyaline, postmarginal vein not more than $2.3 \times$ as long as stigmal vein, fore wing with basal area bare, petiole cylindrical and rugose, lateral margins of petiole rounded (not carinate), $2.1-3.8 \times$ as long as broad in females, $8.3-10 \times$ as long as broad in males, and smooth antecostal sulcus.

\section{Key}

In the Burks et al. (2018) species group key, this species group keys to '[undescribed set of Neotropical species]' in the Orasema festiva couplet with more than 6 digits, although we now recognise that some specimens have as few as 4 digits. All festiva group species have a lateral carina on the petiole, which is absent in the lasallei group.

\section{Diversity}

We recognise four species in the group, Orasema janzeni (Costa Rica and Nicaragua), O. lasallei (Costa Rica, Honduras, Mexico, Nicaragua), O. peckorum (Brazil), and O. vasquezi (Colombia).

Biology. Orasema lasallei was taken from a nest of Pheidole vorax (Fabr.) (Myrmicinae).

\section{Key to species of the $\boldsymbol{O}$. Iasallei species group}

1. Frons vertically costate, lower face mostly smooth (Figure 3a, 4e); labrum with 6-12 digits (Figure 3a,b) ................................................................................................................ 2

Frons rugulose or reticulate, lower face with some sculpture (Figure 3f); labrum with 4-8 digits (Figure $3 \mathrm{f}$ ).

2. Hind femur yellow (Figure 3c, d).................................................. Orasema lasallei sp. nov. Hind femur dark brown medially (Figure 4d) ........................... Orasema vasquezi sp. nov.

3. Labrum with $8(+)$ or $6(\widehat{\jmath})$ digits; frons rugulose-reticulate; basal area of speculum bare; postmarginal vein $2.2 \times$ as long as stigmal vein Orasema peckorum sp. nov. Labrum with 4-6 digits; frons finely reticulate; basal area of speculum closed by broad band of setae; postmarginal vein $1.4-2.2 \times$ as long as stigmal vein. 
Orasema lasallei sp. nov. (lasallei species group)

(Figures 1, 2, 3a-d)

http://zoobank.org/urn:Isid:zoobank.org:act:DFB5A4A8-6CC0-4C1F-8B53-

09B8A062C652

\section{Diagnosis}

This species is recognised by the antenna with 8 funiculars, basal area and speculum of fore wing bare, basal third of hind wing with sparse microsetae, face generally smooth but with the frons vertically costate, labrum with 6-11 marginal digits (Figure 3a,b), clypeus slightly rounded and projecting ventrally and with a distinct anteclypeus. As well, the head and mesosoma are blue green to darkly violaceous, the scape and legs yellow, and the gaster yellowish-brown with dark brown maculations dorsally. Of the specimens where it could be observed, the variation in labral digits ranged from 6 (1 specimen), 7 (5), 8 (7), 9 (5), 10 (3), to 11 (1).

Female. Length 3.1-4.9 mm.

Colour: Head and mesosoma blue green, metallic green, or darkly violaceous. Scape yellow; pedicel and anellus yellowish-brown; flagellum dark brown. Mandible yellowishbrown with dark brown margins and teeth; maxilla and labium yellow to pale brown. Coxae same colour as body or dark metallic purple. Fore wing hyaline; venation yellowish

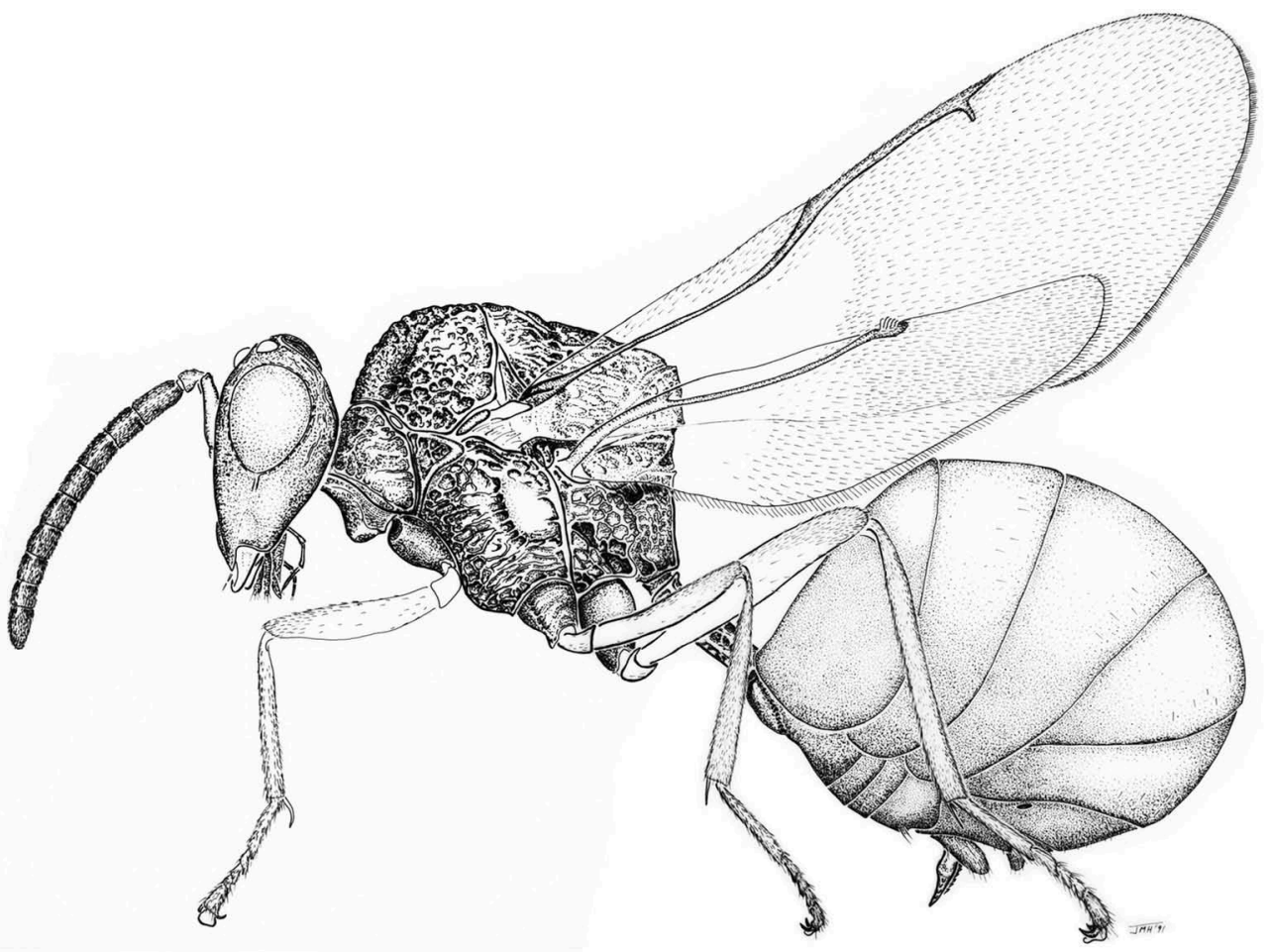

Figure 1. Orasema lasallei sp. nov. 

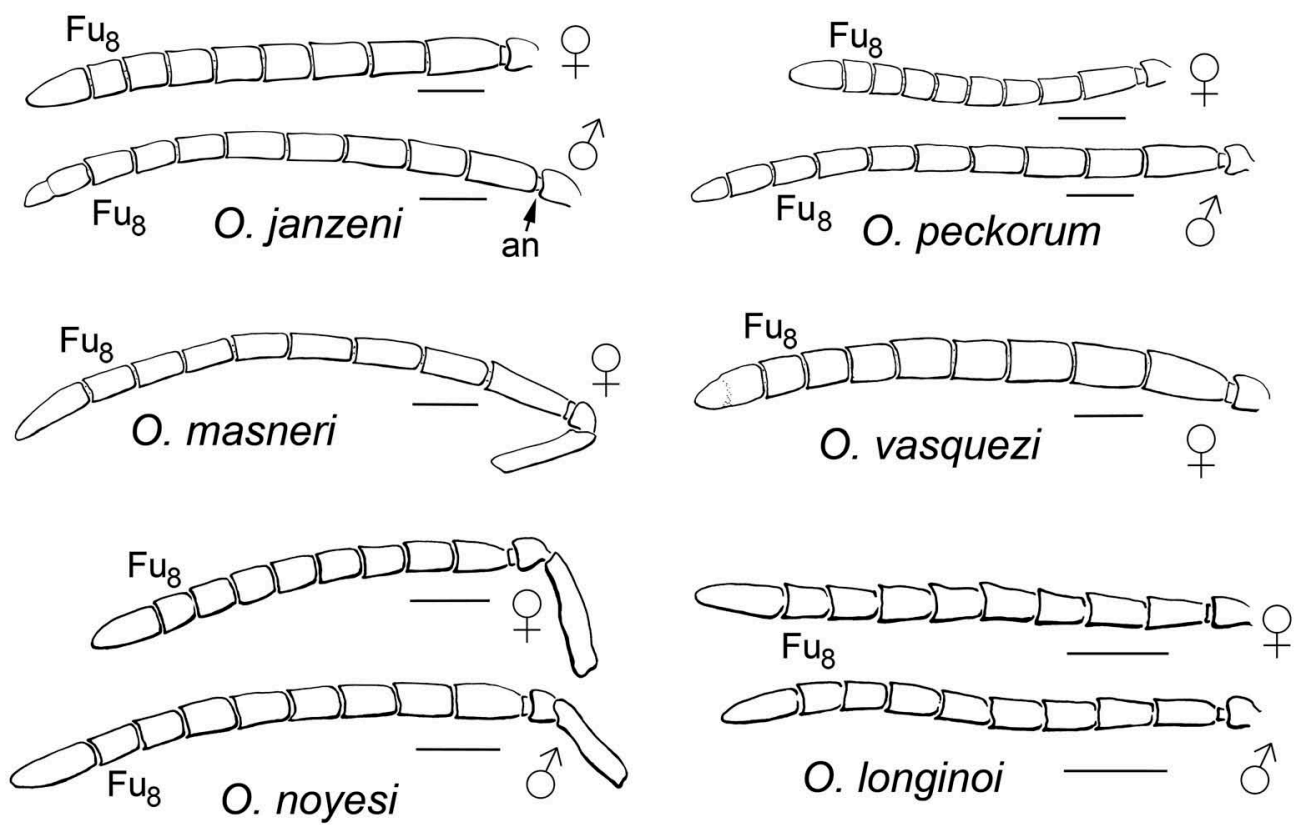

Figure 2. Antennae. an, anellus; $\mathrm{Fu}_{8}$, funicular 8 . Scale bar $=0.25 \mathrm{~mm}$.

to pale brown. Petiole same as mesosoma; gaster yellowish-brown, $\mathrm{Mt}_{1}$ brown with posterior band of yellow, remaining tergites yellow with dorsal brown band.

Head in frontal view subtriangular; $1.1-1.3 \times$ as broad as high; face fairly smooth, frons vertically costate and setose, lower face adjacent to clypeus smooth and setose (Figure 3a); scrobal depression deep, laterally rounded, with transverse carinae and a vertical median ridge; dorsal scrobal depressions absent; eyes bare, inter-ocular distance 1.4-1.8x eye height; malar space 0.7-0.9× eye height; supraclypeal area slightly longer than broad, smooth with weak sculpture along lateral margins; clypeus fairly smooth with short setae; epistomal sulcus vaguely defined and shallowly impressed; anterior tentorial pit strongly impressed; anteclypeus present, ventral margin of clypeus slightly rounded. Labrum with 6-11 digits. Mandibular formula 3:2 or 3:3. Palpal formula 3:3. Occiput aciculate, shallowly emarginate in dorsal view, dorsal margin abrupt; temples broad. Scape not reaching median ocellus. Pedicel small and globose. Flagellum with 8 funiculars; flagellum length 1.3$1.4 \times$ head height; F2 broader than pedicel, $1.5-2.6 \times$ as long as broad, 1.1-1.2x as long as F3; following funiculars subequal in length, equal in width; clava subconical.

Mesosoma 1.0-1.3x as long as high. Mesoscutal midlobe rugose-reticulate to rugoseareolate, bare; lateral lobe coarsely rugose-areolate for the most part; notauli deep. Axilla rugose-areolate, dorsally rounded, on roughly same plane as scutellum; scutoscutellar sulcus broad, regularly foveate, reaching transscutal articulation medially; scutellar disc as long as or slightly longer than broad, mesoscutellar disc coarsely rugose-areolate; frenal line irregularly foveate; frenum rugose-areolate; axillular sulcus absent to very weakly margined; axillula strongly rugose-areolate. Propodeal disc broadly rounded, without depression or carina; shallowly areolate; callus rugose, with a few small setae; callar nib present or absent. Prepectus triangular dorsally, strongly narrowed ventrally, sculpture 


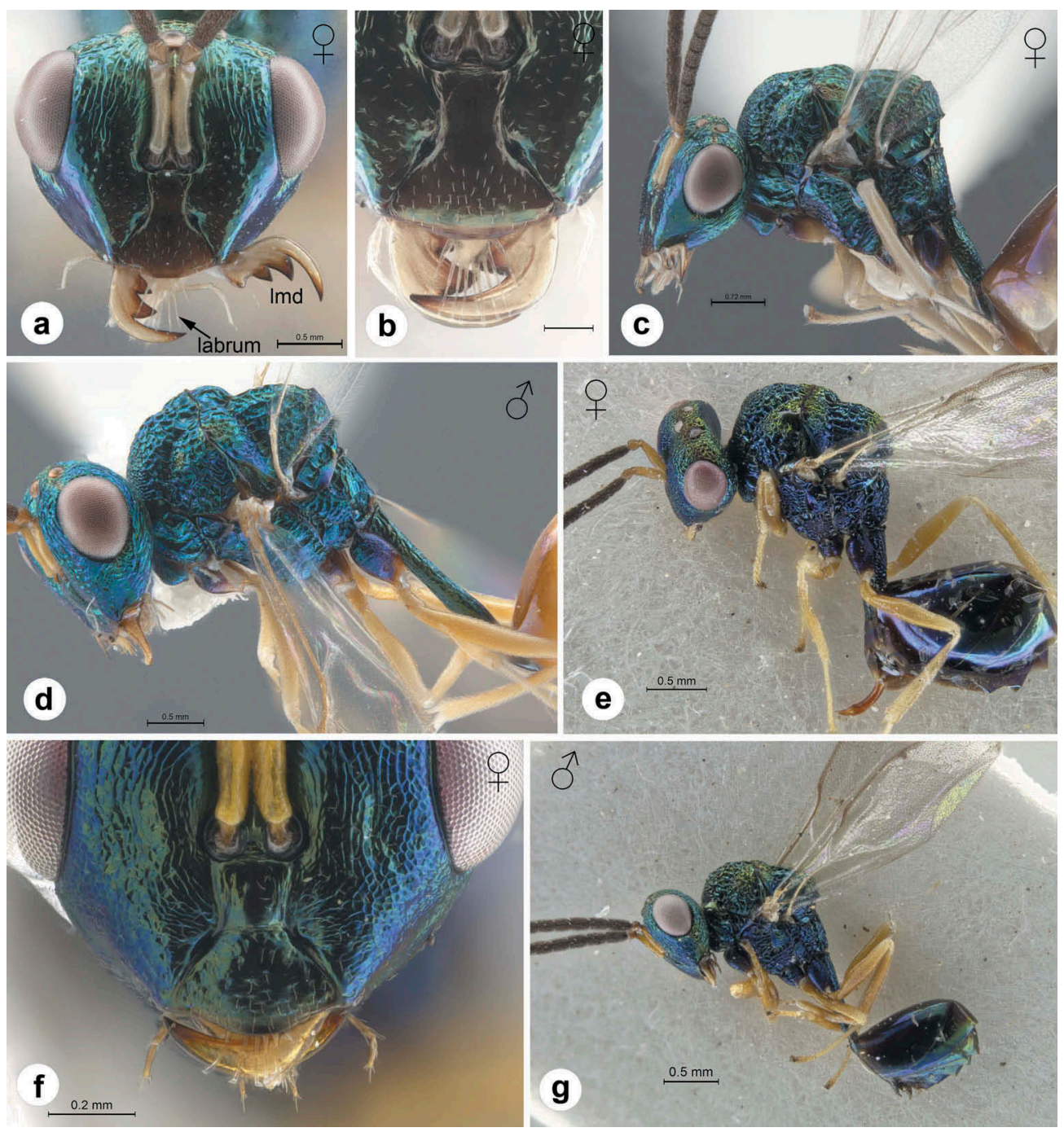

Figure 3. (a-c) Orasema lasallei sp. nov. $q$ holotype: (a) head; (b) mouthparts (c) head and mesosoma. (d) O. lasallei $\hat{O}$, head and mesosoma. (e-f) Orasema janzeni sp. nov. + holotype: (e) head and mesosoma; (f) lower face. $(\mathrm{g})$ O. janzeni ${ }^{3}$ : habitus.

rugose. Mesepisternum areolate laterally, smooth ventrally, broadly rounded anterior to mid coxa. Upper mesepimeron smooth and weakly costate to costate; lower mesepimeron costate; transepimeral sulcus weakly impressed. Metepisternum laterally nearly smooth. Propleuron convex, weakly reticulate. Postpectal carina weak. Hind coxa 1.4-$1.9 \times$ as long as broad, weakly reticulate dorsally, becoming smooth ventrally; hind femur 4.7-6.3 $\times$ as long as broad, with short, dense setae apically; hind tibia densely setose. Fore wing 2.5-2.7× as long as broad, 2.6-2.7× as long as mesothorax; basal cell and speculum bare, costal cell and wing disc densely setose; marginal fringe relatively long; submarginal vein with small setae; marginal vein with dense short setae; stigmal vein 1.4-3.0× as long as broad, slightly angled; uncus weak and blunt; stigma with 3 sensilla in a straight line; 
postmarginal vein $1.3-3.6 \times$ as long as stigmal vein. Hind wing pilose apically, basal third microsetose but appearing bare, costal cell mostly bare except for several setae in extreme apex.

Metasoma: Petiole cylindrical, linear in profile, $2.1-3.3 \times$ as long as broad, $1.1-1.4 \times$ as long as hind coxa, rugose-areolate, anterior carina strong, lateral margin rounded, ventral sulcus absent. Antecostal sulcus crossed by minute carinae; acrosternite posteriorly rounded to weakly angulate; apical setae of hypopygium with four long hairs clustered on each side of midline. Ovipositor slightly curved cephalad; subapical carina weak; first (ventral) valvula with 3-4 strong, widely separated teeth, second (dorsal) valvula with 6-7 annuli that are broadly separated.

Male. Length $3.46 \mathrm{~mm}$. Head width $1.2 \times$ head height; scape yellow; flagellum with 8 funiculars, clava 2-segmented, flagellum length $1.2 \times$ head height, anellus minute, difficult to distinguish, F2 $2.6 \times$ as long as broad, hind femur yellow; petiole $10 \times$ as long as broad, $2.9 \times$ as long as hind coxa.

Ant Host: Two teneral adults (wings in process of unfolding) taken from nest of Pheidole vorax (Fabr.) (Myrmicinae).

DNA Matrix Name (Baker et al. 2020): Orasema_nsp_"dus"_CRI_D4715.

Phylogenetic Position (Baker et al. 2020): This species has variable placement in different analyses: as sister group to the susanae group in the Anchored Hybrid Enrichment (AHE) results (348 loci) or the combined Sanger and AHE results; or as sister to the straminiepes group in the Sanger Sequencing results. In all results, it is placed as a unique branch and is never included within any of the other species groups. Notably, it never groups with the festiva species group, which also have numerous digits on the labrum, suggesting that the multidigitate labrum, versus 4-digitate labrum, arose at least three times within Orasema.

Holotype: Costa Rica: Guanacaste: Santa Rosa Nat. Pk., 300 m, 1053'33"N, 8545'59"W, 1-22.vi.1985, D.H. Janzen \& I.D. Gauld [1, UCRCENT00161412], deposited in CNC. Paratypes: Costa Rica: Alajuela: 5 km W San Ramon, 1200 m, 1005'14"N, 84³0'49"W vii.1997, O. Castro \& P. Hanson [1 9 , MUCR: UCRCENT00479322]. Guanacaste: P. N. Santa Rosa, $200 \mathrm{~m}, 10^{\circ} 53^{\prime} \mathrm{N}, 85^{\circ} 45^{\prime} \mathrm{W}, 24 . \mathrm{v}-14 . v i .1986, \mathrm{P}$. Hanson [2, MUCR: UCRCENT00479339-

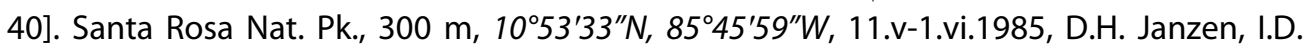
Gauld [1 1 2 2, , UCRC: UCRCENT00408492, UCRCENT00408494, UCRCENT00414048]. Santa

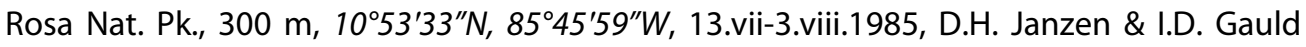
[1 , UCRC: UCRCENT00408493]. Santa Rosa Nat. Pk., 300 m, 1053'33"N, 8545'59"W, 22.vi13.vii.1985, D.H. Janzen \& I.D. Gauld [1, UCRC: UCRCENT00408491]. Santa Rosa NP

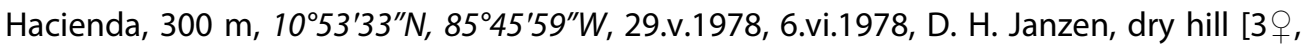
UMNH: UCRCENT00476435]. Limon: Hitoy Cerere Biological Reserve, $150 \mathrm{~m}, 9^{\circ} 40^{\prime} 03^{\prime \prime} \mathrm{N}, 83^{\circ}$ 01'47"W, 13.vi.2015, M. Branstetter, along stream, trop. rainforest, reared Pheidole vorax nest MGB2656 [2ᄋ, UCRC: UCRCENT00446848 (D4715), UCRC: UCRCENT00455978]. San Jose: Ciudad Colón, 800 m, 954'33" $N$, 84¹4'31"W, vi-vii.1990, Luis Fournier [1 9 , USNM: UCRCENT00161411]. San Antonio de Escazu, $1300 \mathrm{~m}, 9^{\circ} 53^{\prime} 00^{\prime \prime} N, 84^{\circ} 08^{\prime} 00^{\prime \prime} W$, 5.vi.1989, P. Hanson [1 9 , UCRC: UCRCENT00408495]. El Salvador: $3 \mathrm{mi}$. W Quezaltepeque, $500 \mathrm{~m}$, $13^{\circ} 49^{\prime} 01^{\prime \prime} N, 89^{\circ} 18^{\prime} 29^{\prime \prime} W, 23 . v i .1961$, M.E. Irwin [1 $ᄋ$, UCDC: UCRCENT00477843]. Honduras: Olancho: Catacamas, $15^{\circ} 50^{\prime} 00^{\prime \prime} \mathrm{N}, 85^{\circ} 51^{\prime} 00^{\prime \prime} \mathrm{W}$, viii.1995, R. Cave, lowland gallery forest, Malaise Trap [1 + , MZLU: UCRCENT00242606]. Mexico: Chiapas: $1 \mathrm{mi}$ S Rayon, $1280 \mathrm{~m}, 17^{\circ}$ $11^{\prime} 25^{\prime \prime} \mathrm{N}, 93^{\circ} 00^{\prime} \mathrm{O}^{\prime \prime} \mathrm{W}, 16$ June 1965, Burks, Meyer, Shaffner [5우, deposited in TAMU: 
UCRCENT00243140, UCRCENT00243142-44, UCRC: UCRCENT00161424]. Veracruz: Orizaba, $1240 \mathrm{~m}, 18^{\circ} 50^{\prime} 43^{\prime \prime} \mathrm{N}, 97^{\circ} 06^{\prime} 14^{\prime \prime} W, 3$. [2+ , MHNG: UCRCENT00412228-29].

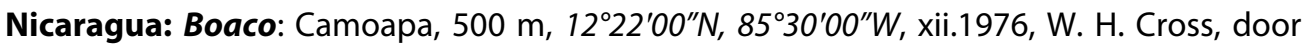
yard Gossypium hirsutum, swp [1 9, MEM: UCRCENT00242503].

\title{
Other material examined
}

Costa Rica: San Jose: San Jose, 1150 m, 9 $9^{\circ} 55^{\prime} 00^{\prime \prime} N, 84^{\circ} 04^{\prime} 00^{\prime \prime} W$, M. Valerio [3, USNM: UCRCENT00416718-19].

Etymology. Named after John La Salle of the Australian National Insect Collection for his amazing work in Chalcidoidea and the Atlas of Living Australia.

\author{
Orasema janzeni sp. nov. (lasallei species group) \\ (Figure 2, 3e-g) \\ http://zoobank.org/urn:Isid:zoobank.org:act:C15B91AF-2A42-4527-948C- \\ 8C3D70BEEBF3
}

\section{Diagnosis}

Recognised by the labrum with 4-6 digits, scape and legs yellow, facial sculpture reticulate, vertex rugulose, mesosoma coarsely rugose-areolate and bluish green with reddish dorsal patches, and fore wing pilose but with the basal area and speculum bare and the speculum open basally, and the hind wing with reduced setation, both in size and numbers, in the basal half. Of the specimens where it could be observed, the variation in labral digits ranged in females from 4 (4 specimens), 5 (3) to 6 (3), and in males from 4 (13), 5 (8) to 6 (8).

Female. Length 3.02-3.8 $\mathrm{mm}$.

Colour: Head and mesosoma blue green. Scape yellow; pedicel and anellus yellowishbrown; flagellum dark brown to black. Mandible yellowish-brown with margins and teeth dark brown; maxilla and labium dark brown. Coxae dark blue; legs beyond coxa yellow but fore leg pale brown subbasally. Fore wing hyaline; venation pale brown. Petiole same as mesosoma; gaster dark brown.

Head in frontal view subtriangular; head width 1.1-1.2x height; face finely reticulate; scrobal depression shallow, laterally rounded, weakly rugulose; eyes bare, inter-ocular distance 1.4-1.7× eye height; malar space 0.6-0.7× eye height; malar depression absent; supraclypeal area about as long as broad, shorter than clypeus, smooth; clypeus weakly sculptured, nearly smooth with scattered setae; epistomal sulcus vaguely defined; anterior tentorial pit strongly impressed; anteclypeus absent, clypeal margin slightly protruding and broadly rounded medially. Labrum with 4-6 digits. Mandibular formula 3:2. Palpal formula 3:3. Occiput aciculate, shallowly emarginate in dorsal view, dorsal margin abrupt; temples broad. Scape not reaching median ocellus. Pedicel subconical, as broad as F1. Flagellum with 8 funiculars; flagellum length 1.2-1.3x head height; anellus disc-shaped; F2 same width as pedicel, $1.6-2.0 \times$ as long as broad, 1.1-1.3x as long as F3; following funiculars subequal in length, equal in width; clava subconical. 
Mesosoma length 1.1-1.2× height. Mesoscutal midlobe scabrous, bare; lateral lobe coarsely rugose-areolate; notauli deep. Axilla rugose-reticulate, dorsally rounded, on roughly same plane as scutellum; scutoscutellar sulcus broad, regularly foveate, narrowly separated from transscutal articulation medially; scutellar disc slightly longer than broad, coarsely rugose-areolate; frenal line coarsely areolate; frenum rugose-areolate; axillular sulcus absent; axillula rugose-areolate. Propodeal disc broadly rounded, without depression or carina, coarsely rugose-areolate; callus areolate-reticulate, bare; callar nib absent. Prepectus triangular dorsally, weakly narrowed ventrally, sculpture rugose-areolate. Mesepisternum rugose-areolate laterally, smooth ventrally, straight anterior to mid coxa. Upper mesepimeron smooth to weakly rugose ventrally; lower mesepimeron rugulose; transepimeral sulcus distinct. Metepisternum laterally rugulose. Propleuron convex, coriaceous. Postpectal carina prominent. Hind coxa $1.4-2.5 \times$ as long as broad, weakly reticulate; hind femur 5-6x as long as broad, with even cover of short, dense setae; hind tibia densely setose. Fore wing 2.3-2.6x as long as broad, 2.2-2.7× as long as mesothorax; basal cell and speculum bare, costal cell and wing disc densely setose; marginal fringe relatively long; submarginal vein with several long setae in basal half, with smaller setae apically; marginal vein with minute setae; stigmal vein 1.5-3.3 $\mathrm{x}$ as long as broad, perpendicular to anterior wing margin; uncus absent; stigmal sensilla indistinct; postmarginal vein $1.4-2.2 \times$ as long as stigmal vein. Hind wing has reduced setation, both in size and numbers, in the basal quarter; costal cell setose apically.

Metasoma: Petiole cylindrical, linear in profile, $1.8-2.7 \times$ as long as broad, $1-1.6 \times$ as long as hind coxa, rugose, anterior carina strong, lateral margin rounded, ventral sulcus absent. Antecostal sulcus irregularly sculptured; acrosternite posteriorly rounded; apical setae of hypopygium with several long hairs on each side of midline. Ovipositor slightly curved cephalad; subapical carina present; first (ventral) valvula with 10-11 small, closely spaced teeth, second (dorsal) valvula with 8-10 annuli that are broadly separated dorsally, carinae weakly coalescing; third valvula separate.

Male. Length 3.17-3.31 mm. Head width 1.1-1.2× height; scape yellow; flagellum with 8 funiculars, clava 1 or 2-segmented, flagellum length 1.6-1.7× head height, anellus disc-shaped, F2 broader than pedicel, $1.7-2.5 \times$ as long as broad; legs beyond coxa yellow. Petiole $6.6-7.0 \times$ as long as broad, $2-2.3 \times$ as long as hind coxa.

Holotype: Costa Rica: Guanacaste: Est. Las Pailas, P.N. Rincon de la Vieja, $800 \mathrm{~m}, 10^{\circ}$ 46'31"N, 85²0'59"W, 12-30.x.1993, D. Garcia [1 9 , INBIOCRI01671288], deposited in INBIO. Paratypes: Costa Rica: Alajuela: P.N. Volcán Tenorio, Sector El Pilón, 700-800 m, 10 42'11"N, 8459'25"W, 11-17.ii.2004, J. Azofeifa, C. Janzen, screen sweep [1 ठ̂, INBIO: INBIOCRI03983195]. Cartago: Monumento Nacional Guayabo, A.C.A.C. Amistad, $1100 \mathrm{~m}$, $9^{\circ} 58^{\prime} 21^{\prime \prime} \mathrm{N}, 83^{\circ} 41^{\prime} 34^{\prime \prime} \mathrm{W}$, vi.1994, G. Fonseca [1 , INBIO: INBIOCRI01882134]. Guanacaste: Est. Las Pailas, P.N. Rincon de la Vieja, 800 m, 1046'31"N, 85²0'59"W, 12-30.x.1993, D. Garcia [8 ${ }^{3} 2$ 2 1?\#, INBIO: INBIOCRI01671306, INBIOCRI01671325, INBIOCRI01671366-74]. Est. Las

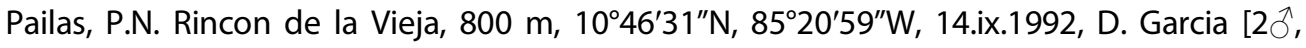
INBIO: INBIOCRI00844929, INBIOCRI00845269]. Est. Las Pailas, P.N. Rincon de la Vieja, $800 \mathrm{~m}$, $10^{\circ} 46^{\prime} 31^{\prime \prime} \mathrm{N}, 85^{\circ} 20^{\prime} 59^{\prime \prime} \mathrm{W}, 19-25 . v i i .1993$, D. Garcia [1, INBIO: INBIOCRI01127372]. Est. Las

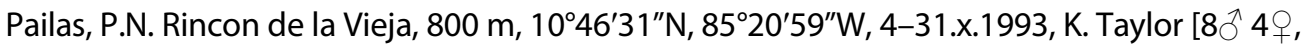
INBIO: INBIOCRI01814111-12, INBIOCRI01814117, INBIOCRI01814133, INBIOCRI01814138, INBIOCRI01814162, INBIOCRI01814197, INBIOCRI01814212, INBIOCRI01814246, INBIOCRIO 
1814289, INBIOCRI01814294, INBIOCRI01814365]. Est. Las Pailas, P.N. Rincon de la Vieja, $800 \mathrm{~m}, 10^{\circ} 46^{\prime} 31^{\prime \prime} \mathrm{N}, 85^{\circ} 20^{\prime} 59^{\prime \prime W}, 5-24 . v i i i .1994$, D.G. Garcia [2خ̂́, INBIO: INBIOCRI01994058, INBIOCRI01994074]. Est. Las Pailas, P.N. Rincon de la Vieja, 800 m, 1046'31"N, 85²0'59"W, 7-19.ii.1994, D.G. Garcia [1 $\sigma^{\prime}$, INBIO: INBIOCRI01986143]. Est. Las Pailas, P.N. Rincon de la Vieja, 800 m, 1046'31"N, 85²0'59"W, viii-ix.1993, G. Rodriguez, J. Sihezar [1 $0^{\circ} 1$ ㅇ, INBIO: INBIOCRI01978884, INBIOCRI01978890]. Est. Pitilla, 9 km S Santa Cecilia, P.N. Guanacaste,

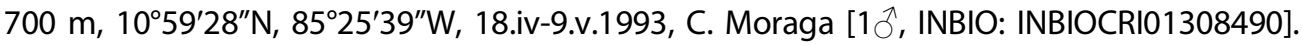

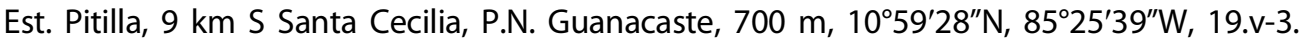
vi.1993, C. Moraga [1 + INBIO: INBIOCRI01341855]. Sector Las Pailas, P.N. Rincon de la Vieja, $800 \mathrm{~m}, 10^{\circ} 47^{\prime} 03^{\prime \prime} \mathrm{N}, 85^{\circ} 21^{\prime} 00^{\prime \prime} \mathrm{W}, 5-17 . v .1994$, Guadalupe Rodriguez [1 $\hat{\sigma}^{\prime}$, INBIO: INBIO CRI02096407]. Heredia: San Joaquin, $10^{\circ} 00^{\prime} 19^{\prime \prime} \mathrm{N}, 84^{\circ} 09^{\prime} 26^{\prime \prime} \mathrm{W}, 1 . x i .1987$, J.A. Ugalde [1 $\hat{O}^{\prime}$ UCRC: UCRCENT00413881]. San Jose: Ciudad Colón, 800 m, 9'54'33"N, 84¹4'31"W, vivii.1990, Luis Fournier [1+, UCRC: UCRCENT00413882]. Santiago de Puriscal, $1005 \mathrm{~m}$, $9^{\circ} 50^{\prime} 47^{\prime \prime} \mathrm{N}, 84^{\circ} 18^{\prime} 51^{\prime \prime} \mathrm{W}$, 8.vii.1963, C.D. Michener et al. [1오, SEMC: UCRCENT00404249]. Nicaragua: Carazo: San Marcos, $11^{\circ} 54^{\prime} 34^{\prime \prime} \mathrm{N}, 86^{\circ} 12^{\prime} 13^{\prime \prime} \mathrm{W}$, Baker [1 $\mathrm{O}^{\prime}$, USNM:

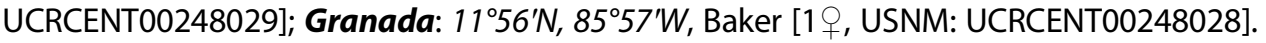

Etymology. Named after Daniel Janzen of the University of Pennsylvania for his amazing support of tropical biology in Costa Rica.

\author{
Orasema peckorum sp. nov. (lasallei species group) \\ (Figures 2, 4a-c) \\ http://zoobank.org/urn:Isid:zoobank.org:act:8C969A68-9200-4453-B0ED- \\ 59E7BB0DE451
}

\title{
Diagnosis
}

Recognised by the labrum with 8 digits, frons rugulose, lower face irregularly rugosereticulate, scape and legs yellow, mesosoma coarsely rugose-areolate and bluish green with reddish patches, and fore wings pilose but with the basal area and speculum bare and the speculum closed basally by a broad band of setae. Basal third of hind wing microsetose, and costal cell with scattered setae in apical half. Closest to O. lasallei and O. vasqueszi, but these have a distinctly costate frons.

Female. Length $4.87 \mathrm{~mm}$.

Colour: Head and mesosoma blue green, darker ventrally; mesosoma with reddish patches on anterior midlobe, axillae, a medial line on scutellar disc, and the frenal line. Scape, pedicel and anellus yellow; flagellum brown. Mandible yellowish-brown with teeth dark brown; maxilla and labium yellowish brown. Fore and mid coxa yellowish-brown, mid coxa with dark brown lateral channel; hind coxa brown dorsally to yellow ventrally; remaining legs yellow. Fore wing hyaline; venation yellowish brown. Petiole blue-green; gaster brown, $\mathrm{Mt}_{1}$ darker brown dorsally and with purplish reflections.

Head in frontal view subtriangular; head width $1.25 \times$ height; frons rugulose to rugosereticulate dorsally, lower face lateral to clypeal area reticulate, upper frons with weak vertical carinae; scrobal depression shallow, laterally rounded, weakly rugulose; eyes bare, 


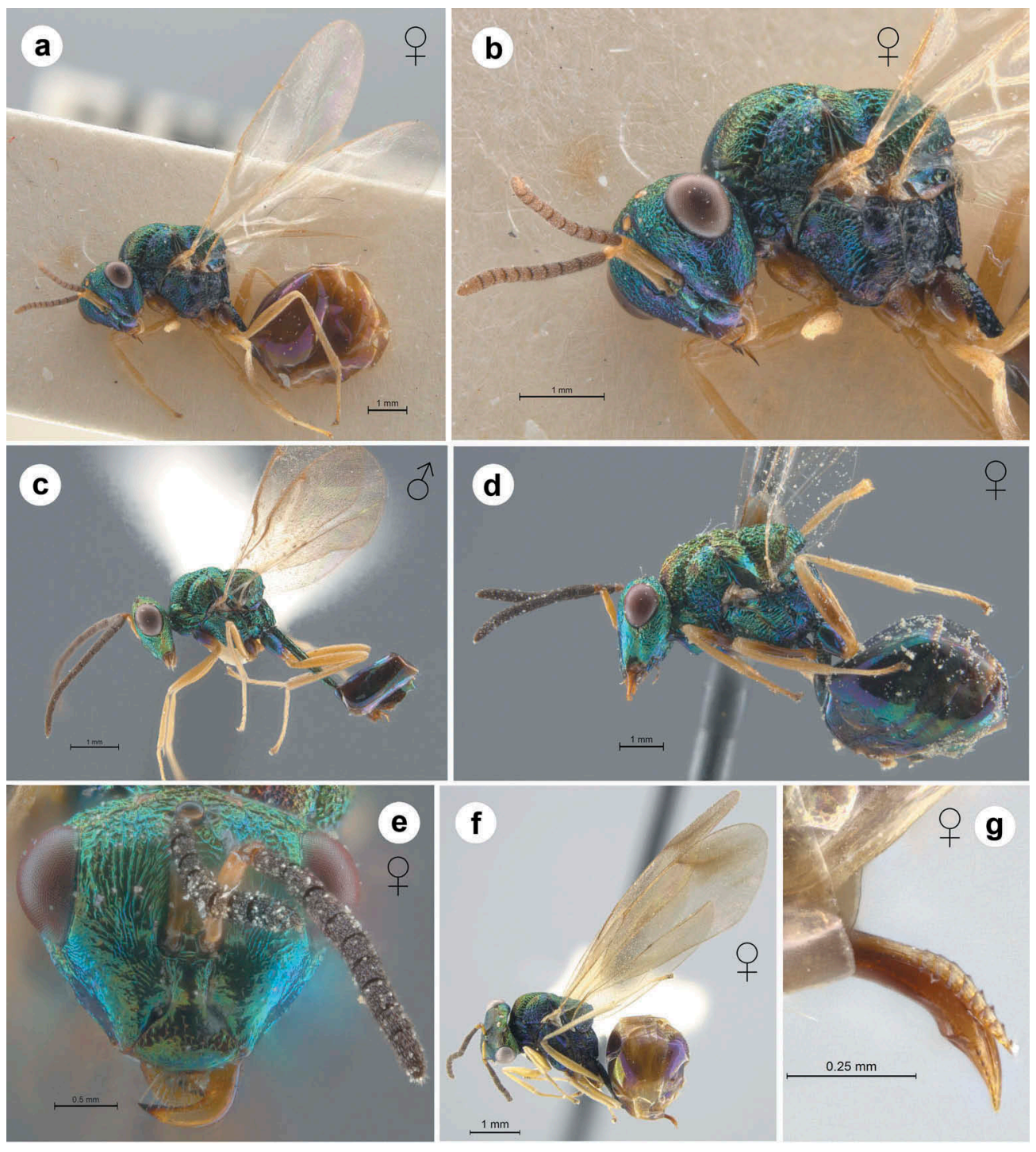

Figure 4. (a-b) Orasema peckorum sp. nov. $q$ holotype: (a) habitus; (b) head and mesosoma. (c) O. peckorum ${ }^{\top}$ : habitus. (d-e) Orasema vasquezi, sp. nov., $q$ holotype: (d) habitus; (e) face. (f-g) O. masneri sp. nov., + holotype: (f) habitus; (g) ovipositor, lateral view.

inter-ocular distance $1.7 \times$ eye height; malar space $0.8 \times$ eye height; supraclypeal area about as long as broad, shorter than clypeus, mostly smooth; clypeus smooth; epistomal sulcus distinct and sharply defined; anterior tentorial pit strongly impressed; anteclypeus weakly differentiated, clypeal margin broadly rounded. Labrum with 8 digits. Mandibular formula 3:2 [?, only partially visible]. Palpal formula 3:3. Occiput aciculate, shallowly emarginate in dorsal view, dorsal margin abrupt; temples broad. Scape not reaching median ocellus. Pedicel globose, broader than F1. Flagellum with 8 funiculars; flagellum length $1.2 \times$ head height; anellus disc-shaped; F2 narrower than pedicel, $2.8 \times$ as long as 
broad, 1.4× as long as F3; following funiculars subequal in length, equal in width; clava subconical.

Mesosoma length 1.0x height. Mesoscutal midlobe rugose-reticulate, more smoothly reticulate anteriorly, bare; lateral lobe areolate-reticulate; notauli deep. Axilla rugosereticulate, dorsally rounded, on roughly same plane as scutellum; scutoscutellar sulcus broad, regularly foveate, broadly separated from transscutal articulation medially; scutellar disc slightly longer than broad, coarsely rugose-areolate; frenal line irregularly foveate; frenum rugulose; axillular sulcus absent; axillula areolate. Propodeal disc broadly rounded, without depression or carina, irregularly rugose-areolate; callus weakly rugulose, bare; callar nib absent. Prepectus triangular dorsally, strongly narrowed ventrally, sculpture reticulate. Mesepisternum areolate-reticulate laterally, smooth ventrally, broadly rounded anterior to mid coxa. Upper mesepimeron mostly smooth; lower mesepimeron reticulate; transepimeral sulcus weakly impressed. Metepisternum laterally irregularly reticulate. Propleuron convex, weakly reticulate. Postpectal carina prominent. Hind coxa $1.8 \times$ as long as broad, completely reticulate; hind femur $6.0 \times$ as long as broad, with short, dense setae apically; hind tibia densely setose. Fore wing $2.6 \times$ as long as broad, $2.5 \times$ as long as mesothorax; basal cell and speculum bare, but speculum closed by setae basally, costal cell and wing disc densely setose; marginal fringe relatively long; submarginal vein with several long setae in basal half, with smaller setae apically; marginal vein pilose; stigmal vein more than $2.5 x$ as long as broad, slightly angled; uncus absent; postmarginal vein $2.2 \times$ as long as stigmal vein. Hind wing pilose apically, basal third micropilose, costal cell with sparse setae in apical half.

Metasoma: Petiole cylindrical, linear in profile, $1.8 \times$ as long as broad, $1.0 \times$ as long as hind coxa, rugose-areolate, anterior carina strong, lateral margin rounded, ventral sulcus present with margins narrowly separated. Antecostal sulcus crossed by minute carinae; acrosternite posteriorly rounded; hypopygium apically with one pair of long setae. Ovipositor not exposed.

Male. Length 4.19-5.21 mm. Head width 1.2-1.3x height; scape yellow, pedicel and anellus pale brown; labrum with 6 digits; flagellum with 8 funiculars, clava 2-segmented, flagellum length $2.1 \times$ height, F2 broader than pedicel, anellus minute, difficult to distinguish, F2 2.3-2.6x as long as broad. Legs beyond coxa yellow. Petiole 8.3-9.3x as long as broad, $2.3-2.6 \times$ as long as hind coxa. Genitalia with long, thin and broadly separated parameres, median process and broad aedeagus.

Holotype: Brazil: Nova Teutonia, 16.xi.1944, F. Plaumann [1 $q$, UCRCENT00309915], deposited in BMNH. Paratypes: Brasil: [M ...] unreadable label, Wthm. [1 $\sigma^{\lambda}, \mathrm{NMW}$

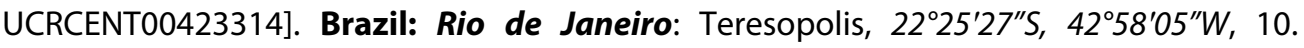

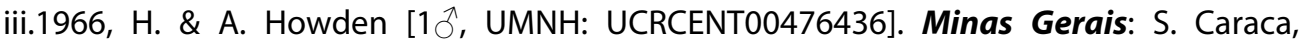

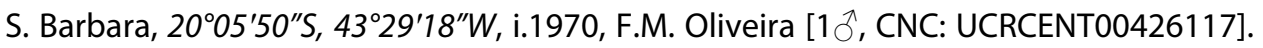

Etymology. Named after Stuart Peck and Jarmila Kukalova-Peck for their outstanding collections and incredible dedication to all that is entomology. 


\author{
Orasema vasquezi sp. nov. (lasallei species group) \\ (Figures 2, 4d,e) \\ http://zoobank.org/urn:Isid:zoobank.org:act:81624AE7-A368-414C-9CCD- \\ E8A9598A4B75
}

\title{
Diagnosis
}

This species is very similar to 0 . lasallei with the frons vertically costate, labrum 12 digitate, and basal area of the wing with similar reduced setation; however in $O$. vasquezi the femora and labiomaxillary complex are brown, the gaster is dark brown with iridescence, and the petiole is slightly longer $(1.2 \times)$ relative to the hind coxa.

Female. Length $5.75 \mathrm{~mm}$.

Colour: Head and mesosoma blue green with reddish patches on vertex, anterior mesosomal midlobe, lateral sidelobe, axillae and frenal line; ventral mesosoma dark bluish green. Scape yellow; pedicel dark brown; anellus brown; flagellum black. Mandible brown with darker margins and teeth; maxilla and labium brown. Coxae dark blue; Fore wing hyaline; venation pale brown. Petiole dark bluish green; gaster black with blue-purple reflections.

Head in frontal view subtriangular; head width $1.2 \times$ head height; face mostly smooth, upper frons vertically costate to rugose laterally and dorsally; scrobal depression deep, laterally rounded, with transverse costae; dorsal scrobal depressions absent; eyes bare; inter-ocular distance $2.2 \times$ eye height; malar space $1.2 \times$ eye height; supraclypeal area longer than broad, mostly smooth; clypeus smooth with shallow setose umbilicate punctures; epistomal sulcus vaguely defined; anterior tentorial pit strongly impressed; anteclypeus absent, ventral margin of clypeus broadly rounded. Labrum with 12 digits. Mandibular formula 3:2 [?, mostly hidden]. Palpal formula 3:3. Occiput aciculate, sharply emarginate in dorsal view with abrupt dorsal margin; temples broad. Scape not reaching median ocellus. Pedicel subconical, as broad as F1. Flagellum with 8 funiculars; flagellum length $1.3 \times$ head height; F2 broader than pedicel, $1.8 \times$ as long as broad, $1.2 \times$ as long as F3; following funiculars subequal in length, equal in width; clava subconical.

Mesosoma length $1.3 \times$ height. Mesoscutal midlobe coarsely rugose-areolate, bare; lateral lobe coarsely rugose-areolate; notauli deep. Axilla rugose-areolate, dorsally rounded, on roughly same plane as scutellum; scutoscutellar sulcus broad, regularly foveate, narrowly separated from transscutal articulation medially; scutellar disc slightly longer than broad, coarsely rugose-areolate; frenal line irregularly foveate; frenum rugose-areolate; axillular sulcus absent; axillula areolate. Propodeal disc broadly rounded, without depression or carina, rugose-areolate; callus finely areolate-reticulate, bare; callar nib absent. Prepectus triangular dorsally, strongly narrowed ventrally, sculpture rugose-areolate. Mesepisternum irregularly areolate-reticulate laterally, smooth ventrally, broadly rounded anterior to mid coxa. Upper mesepimeron smooth with longitudinal costae; lower mesepimeron longitudinally costate; transepimeral sulcus weakly impressed. Metepisternum laterally smooth. Propleuron convex, coriaceous. Postpectal carina prominent. Hind coxa $1.9 \times$ as long as broad, smooth but weakly reticulate basolaterally; hind femur $6.0 \times$ as long as broad, with short, dense setae apically; hind tibia densely setose. Fore wing $2.6 \times$ as long as broad, $2.6 \times$ as long as mesothorax; basal cell and speculum bare, costal cell and wing disc densely setose; marginal fringe relatively long; submarginal vein with small setae; marginal vein pilose; 
stigmal vein $4.3 \mathrm{X}$ as long as broad, very thin, perpendicular to anterior wing margin; postmarginal vein $3.4 \times$ as long as stigmal vein. Hind wing pilose apically, basal third micropilose, costal cell mostly bare except for several setae in extreme apex.

Metasoma: Petiole cylindrical, linear in profile, $3.0 \times$ as long as broad, $1.2 \times$ as long as hind coxa, rugose-areolate, anterior carina strong. Antecostal sulcus smooth; acrosternite posteriorly rounded; apical setae of hypopygium with medial patch of short setae. Ovipositor not exposed.

Male. Unknown.

Holotype: Colombia: Pensilvania: Caldas, $1500 \mathrm{~m}, 5^{\circ} 22^{\prime} 00^{\prime \prime} \mathrm{N}, 75^{\circ} 05^{\prime} 00^{\prime \prime} \mathrm{W}, 25 . v i .1978, \mathrm{~J}$. H. Cane [1 9 , UCRCENT00404251], deposited in SEMC.

Etymology. Named after Aymer Andrés Vásquez-Ordóñez, a colombian student of the Eucharitidae.

\section{Orasema wayqecha species group}

\section{Diagnosis}

The following changes to the diagnosis provided by Herreid and Heraty (2017) are made: postgena expanded to cover the maxillary complex or open exposing the maxillary complex, labrum with 6-10 digits, face mostly smooth and shining or reticulate, clypeus longer than broad or broader than long. With these changes, the wayqecha group is still easily diagnosed from all other species groups by containing the only Orasema species with maculations on the fore wings. Additionally, wayqecha group has entirely yellow femora, a broadly triangular face, elongate fore coxa, and fore wings over three times the length of the mesosoma.

\section{Key}

Changes to the key provided in Herreid and Heraty (2017) to accommodate the new species are provided below. In the Burks et al. (2018) species group key, O. masneri keys to 'undescribed Neotropical species' in couplet 8.

\section{Diversity}

Three species are recognised in this group: Orasema masneri sp. nov. (Ecuador), Orasema quadrimaculata Herreid \& Heraty (Colombia) and Orasema wayqecha Herreid \& Heraty (Peru).

\section{Biology}

Orasema wayqecha is known to oviposit near the extrafloral nectaries of two species in the family Primulaceae, and larvae and pupae have been recovered from the nests of Pheidole alfaroi Emery (Herreid and Heraty 2017). 
1. Fore wing with dark spots at least along cubital fold, fore wing at least $3 \times$ longer than mesosoma. All femora yellow. Labrum with 6-10 digits. Postmarginal vein at least $4 \times$ longer than stigmal vein. Flagellum with 8 funiculars and at least $1.75 \times$ the length of the head (wayqecha group) 2 Characters not matching the above Orasema species groups

2. Face entirely reticulate. Postgena widely spaced, exposing maxillary complex basally. Labrum with 6 digits Orasema masneri sp. nov. Face mostly smooth with at most scattered punctures or longitudinal striations near eye. Postgena closely spaced, hiding the maxillary complex basally. Labrum with 7-10 digits

3. Fore wing of female with three to four darkened areas, post-stigmal pigmentation not reaching wing apex and sometimes very light, wing spots absent or barely visible in male. Labrum with 7-8 digits. Frons with vertical and often distinct striae medial to eye Orasema wayqecha Herreid \& Heraty Fore wing with four darkened areas in both male and female, post-stigmal pigmentation reaching wing apex. Labrum with 9-10 digits. Frons weakly rugose medial to eye Orasema quadrimaculata Herreid \& Heraty

\author{
Orasema masneri sp. nov. (wayqecha species group) \\ (Figures 2, 4f,g) \\ http://zoobank.org/urn:Isid:zoobank.org:act:5FA765F8-FE63-4986-8D94- \\ 9CC086CA957B
}

\title{
Diagnosis
}

Orasema masneri can be recognised by having the basal area of the fore wing bare, face finely reticulate, clypeus broadly rounded and projecting ventrally without a differentiated anteclypeus, and maculated fore wings. As well, the head and mesosoma are blue green, the scape and legs yellow, and the gaster yellowish brown with dark brown maculations dorsally.

Female. Length 4.19-4.31 $\mathrm{mm}$.

Colour: Head and mesosoma blue-green with blue and copper patches, ventral mesosoma dark bluish purple with metallic purple reflections. Scape yellow; pedicel and anellus yellow; flagellum brown. Mandible yellow with brown margins; maxilla and labium yellow. Coxae yellow medially and dark purple-brown laterally, remainder of leg yellow. Fore wing with a distinct infuscate patch posterior to stigmal vein and irregular faint infuscate patches over entire wing; venation yellowish brown. Petiole same as mesosoma; gaster brown, $\mathrm{Mt}_{1}$ and apical tergites dark brown.

Head in frontal view subtriangular; width $1.2 \times$ height; face reticulate; scrobal depression shallow, laterally rounded, reticulate; dorsal scrobal depressions absent; eyes bare, inter-ocular distance 1.8-1.9x eye height; malar space $0.8-0.9 \times$ eye height; supraclypeal area longer than broad, smooth medially; clypeus smooth to weakly sculptured; epistomal sulcus narrow but shallow; anterior tentorial pit shallow; anteclypeus weakly differentiated, clypeal margin broadly rounded. Labrum with 6 digits. Mandibular formula 3:2. 
Palpal formula 3:3. Occiput aciculate, emarginate in dorsal view, dorsal margin abrupt with sharp dorsal margin. Scape almost reaching median ocellus. Pedicel globose, broader than F1. Flagellum with 8 funiculars; flagellum length $1.7 \times$ head height; F2 $3.1-4.2 \times$ as long as broad, 1.2-1.4X as long as F3; following funiculars subequal in length, equal in width; clava subcylindrical.

Mesosoma length 1.2-1.3× height. Mesoscutal midlobe rugose-reticulate, bare; lateral lobe finely rugose-areolate; notauli deep. Axilla finely rugose-areolate, dorsally rounded, on roughly same plane as scutellum; scutoscutellar sulcus narrow, regularly foveate, reaching transscutal articulation medially; scutellar disc slightly longer than broad, areolate; frenal line irregularly foveate but present; frenum rugose-areolate; axillular sulcus absent to weakly margined; axillula with subparallel longitudinal carinae connected by small perpendicular carinae. Propodeal disc broadly rounded, without depression or carina that is barely visible, rugose-areolate; callus weakly areolate-reticulate, with a few small setae; callar nib absent. Prepectus triangular dorsally, strongly narrowed ventrally, sculpture rugose. Mesepisternum weakly rugose-areolate laterally, smooth ventrally, broadly rounded anterior to mid coxa. Upper mesepimeron smooth with longitudinal costae; lower mesepimeron mostly smooth; transepimeral sulcus distinct. Metepisternum laterally rugose-reticulate. Propleuron convex, weakly reticulate. Postpectal carina weak. Hind coxa 1.5-1.8x as long as broad, reticulate dorsally, becoming smooth ventrally; hind femur $6.9-10.5 \times$ as long as broad, with even cover of long semi-erect setae, basally mostly bare with a few semi-erect setae; hind tibia densely setose. Fore wing $2.8-3.4 \times$ as long as broad, 3.4-3.7x as long as mesothorax; entirely setose, wing disc densely setose, basal area of basal cell bare; marginal fringe relatively long; submarginal vein with dense erect setae in basal half, apical half and marginal vein pilose; stigmal vein 1.5-2.0x as long as broad, perpendicular to anterior wing margin; postmarginal vein $4.1-4.4 \times$ as long as stigmal vein. Hind wing entirely pilose.

Metasoma: Petiole cylindrical, linear in profile, $2.7-3.4 \times$ as long as broad, $1.4-1.6 \times$ as long as hind coxa, rugose, anterior carina strong. Antecostal sulcus with shallow sculpture; acrosternite weakly angulate posteriorly. Apical setae of hypopygium with two pairs of short setae on each side of midline. Ovipositor slightly curved cephalad; subapical carina weak; first (ventral) valvula with 5-6 strong, widely separated teeth, second (dorsal) valvula with 8-10 annuli that are broadly separated dorsally by smooth area.

Male. Unknown.

Holotype: Ecuador: Napo: 5 km S Baeza, 1700 m, 0²9'00"S, 7752'00"W, 13.ii.1983, Masner \& Sharkey [1+, UCRCENT00161413], deposited in CNC. Paratypes: same locality and date [1 1 1 1 , CNC: UCRCENT00161415, UCRCENT00426113].

Etymology. Named after Lubomir Masner of the Canadian National Collection for his unique ability to pass along the enthusiasm for Hymenoptera and collecting to an entire generation. 


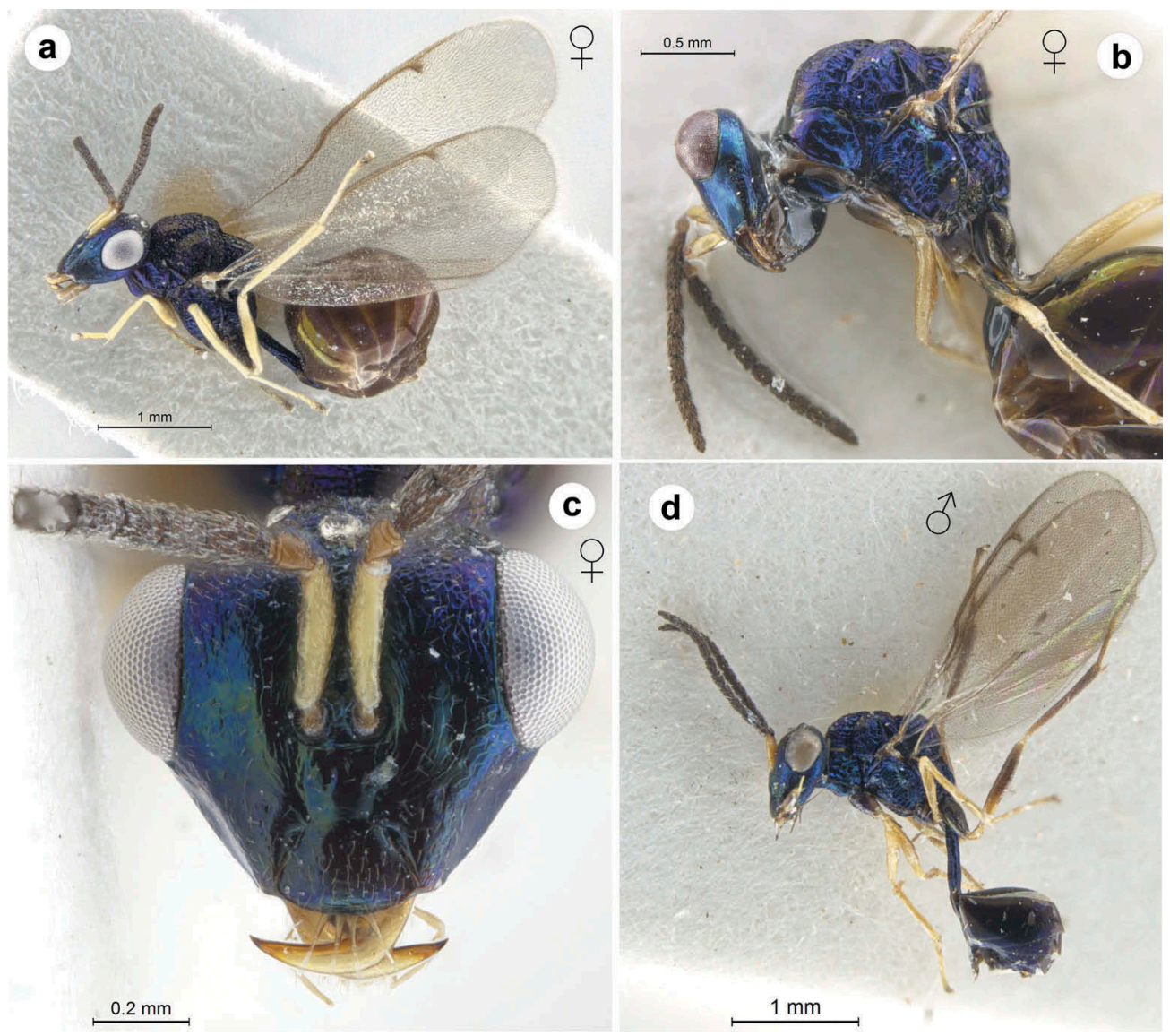

Figure 5. (a-c) Orasema longinoi sp. nov. $q$ holotype: (a) habitus; (b) head and mesosoma; (c) face. (d) O. longinoi $\widehat{O}$ : habitus.

Orasema longinoi sp. nov. (unplaced to species group)

(Figures 2, 5a-d)

http://zoobank.org/urn:Isid:zoobank.org:act:B86AFE78-7C48-4092-BC46-

84D2CEB90DD2

\section{Diagnosis}

Within Orasema, recognised by the combination of the labrum 4-digitate, antennal flagellum with $8\left(+\right.$ ) or $9\left({ }^{\Uparrow}\right)$ funiculars (Figure 2), facial sculpture nearly smooth, mesoscutum and mesoscutellum scabrous to strongly rugose-areolate, specular area pilose with sparse basal setation, fore wing slightly infuscate, and hind wing densely pilose. Females all have the legs beyond the coxa entirely yellow, whereas males have most of the femora and usually all of the hind tibia brown. Molecular analyses consistently place this as a unique lineage that is close to a combination of the festiva, stramineipes and susanae species groups (Baker et al. 2020). The festiva group all have more than 8-11 labral digits, whereas both the straminiepes and susanae groups have 4 or rarely up to 6 labral digits. 
Only the susanae group has 9 funiculars in males. Based on the variable molecular support, never being placed within any of our recognised species groups, and the unique morphology of the species, we consider this as unplaced within Orasema, but related to the groups just mentioned.

Key. In the Burks et al. (2018) species group key, this species keys to '1 currently undescribed Neotropical species' in couplet 6 along with the susanae group. Orasema longino is more strongly scuptured on the mesosoma versus evenly and finely reticulate in the susanae group.

Female. Length 3.02-3.75 $\mathrm{mm}$.

Colour: Head dark blue, mesosoma dark blue with violaceous reflections. Scape yellow; pedicel and anellus brown; flagellum brown. Mandible yellow with brown ventral margins; maxilla and labium light brown. Coxae dark brown with violaceous iridescence; legs beyond coxa yellow. Fore wing slightly infuscate, may be darker along impressions of cubital and basal folds; venation brown. Petiole same as mesosoma; gaster dark brown with violaceous iridescence.

Head in frontal view subtriangular; head width 1.1-1.8x height; upper frons weakly reticulate, lower face smooth or at most with weak surface sculpture; scrobal depression with vague parallel channels, with transverse carinae and vertical median ridge; eyes bare; inter-ocular distance 1.5-1.6x eye height; malar space $0.7 \times$ eye height; supraclypeal area longer than broad, smooth; clypeus smooth and pilose; epistomal sulcus narrow but shallow; anterior tentorial pit strongly impressed; anteclypeus weakly differentiated, clypeal margin very slightly rounded. Labrum with 4 digits (Figure 5c). Mandibular formula 3:2. Palpal formula 3:2. Occiput aciculate, shallowly emarginate in dorsal view, dorsal margin abrupt; temples present, rounded. Scape not reaching median ocellus. Pedicel subconical, as broad as F1. Flagellum with 8 funiculars; flagellum length 1.4-$1.6 \times$ head height; anellus disc-shaped; F2 1.6-2.5x as long as broad, 1.2-1.3x as long as F3; following funiculars subequal in length, equal in width; clava subcylindrical.

Mesosoma length 1.2-1.4× height. Mesoscutal midlobe scabrous dorsally, sparsely setose; lateral lobe coarsely rugose-areolate; notauli deep. Axilla rugose-areolate to scabrous, dorsally well above scutellum; scutoscutellar sulcus broad, irregularly foveate, broadly separated from transscutal articulation medially; scutellar disc as long as broad, coarsely rugose-areolate with broad median depression; frenal line irregularly foveate; frenum rugose-areolate; axillular sulcus distinct and foveate; axillula reticulate. Propodeal disc broadly rounded, rugose-areolate, without depression or carina; callus rugulose, with several long hairs. Prepectus rugose-areolate. Mesepisternum rugose-areolate laterally, smooth ventrally, straight anterior to mid coxa; postpectal carina prominent. Upper mesepimeron smooth; lower mesepimeron smooth with vague sculpture; transepimeral sulcus distinct. Metepisternum laterally rugose. Propleuron convex, coriaceous. Hind coxa 2.0-2.4x as long as broad, reticulate dorsally, becoming smooth ventrally; hind femur 7.0-7.2x as long as broad, apical half with scattered short setae; hind tibia densely setose. Fore wing 2.5-2.7× as long as broad, $2.1-2.6 \times$ as long as mesothorax; basal cell sparsely pilose, costal cell, specular area and wing disc densely setose, anterior distal region of basal cell setose, and cubital vein setose; marginal fringe relatively long; submarginal vein with several long setae in basal half, with smaller setae apically; marginal vein pilose; stigmal vein $1.5-2.0 \times$ as long as broad, slightly angled; uncus present and blunt; 
postmarginal vein $4.3-5.0 \times$ as long as stigmal vein. Hind wing including costal cell entirely pilose.

Metasoma: Petiole cylindrical, linear in profile, $3.4-4 \times$ as long as broad, $1.4-1.5 \times$ as long as hind coxa, rugose, anterior carina strong, lateral margin rounded, ventral sulcus absent. Antecostal sulcus smooth; acrosternite posteriorly rounded. Apical setae of hypopygium present, minute. Ovipositor not exposed.

Male. Length 2.58-3.21 mm. Pedicel, anellus and apical half to two-thirds of hind femur dark brown; hind tibia dark brown or yellow; fore wing slightly infuscate and venation dark brown to black. Head width 1.1-1.3x head height; frons and lower face excluding clypeal region more strongly sculptured with rugose patterns and fine reticulate surface sculpture; flagellum length 1.5-2.2× head height, with 9 funiculars, F2 1.6-2.5× as long as broad; petiole $6.5-9.3 \times$ as long as broad, 2.2-2.8x as long as hind coxa.

Ant Host: Related species collected in nest of Pheidole dossena Wilson (Myrmicinae) (see discussion below).

Variation: There is little variation in the type material other than the wing venation being slightly lighter in colour for the female from Guanacaste (UCRC00312148). Notably, the fore wings have the specular area entirely pilose. Males in the type series differ only in the colouration of the hind tibia, with one of the two males from the same locality (INBIOCRI01793753) having a yellowish-brown instead of dark brown hind tibia. Two male specimens were excluded and likely represent a different species that is recognised primarily by having a bare speculum and a proportionally longer antennal flagellum that is 1.9-2.0x as long as head height. The specimen from Costa Rica with the Pheidole host record has a slightly thicker, black antennal flagellum, the head and mesosoma more strongly sculptured, speculum bare, fore wing hyaline, and hind tibia yellow. The right antenna is still encased by the pupal exuvium. The Florida specimen is very similar but has a pale brown antennal flagellum. The Florida record seems odd as it would represent a very disjunct distribution, but there was a practising entomologist named Carl Stegmaier that lived in what is now Miami, so the record cannot be discounted.

DNA Matrix Name (Baker et al. 2020): Orasema_"AECRI4"_CRI_AE_D4202.

Phylogenetic Position (Baker et al. 2020): This species has variable placement in different analyses: as sister group to the festiva and straminiepes groups in the Anchored Hybrid Enrichment results (348 loci; susanae group and $O$. longinoi not included); as sister to a clade that includes the festiva, straminiepes, and susanae groups, and $O$. longinoi, in the Sanger Sequencing results and also in the combined Sanger and AHE results. In all results, it arises as a unique branch and is never included in any of the other species groups. This species also stands in isolation, in that we have not discovered any other morphologically similar species.

Holotype: Costa Rica: Guanacaste: Guanacaste N.P., Pitilla Biological Station, $10^{\circ}$ 59'31"N, 85²8'20"W, 1.ii.1995, L. Masner, screen sweep, CR-02 [1 +, UCRCENT00312148], deposited in CNC. Paratypes: Costa Rica: Guanacaste: Est. Pitilla, 9 km S Santa Cecilia, P.N. Guanacaste, $700 \mathrm{~m}, 10^{\circ} 59^{\prime} 28^{\prime \prime} \mathrm{N}, 85^{\circ} 25^{\prime} 39^{\prime \prime} \mathrm{W}, 21 . i i i-7 . i v .1993$, P. Rios [2 $0^{\prime}$, INBIO: INBIOCRI01386293, INBIOCRI01386493]. Est. Pitilla, 9 km S Santa Cecilia, P.N. Guanacaste, $700 \mathrm{~m}, 10^{\circ} 59^{\prime} 28^{\prime \prime} \mathrm{N}, 85^{\circ} 25^{\prime} 39^{\prime \prime} \mathrm{W}$, iii.1995, P. Rios, \#4359 [20, INBIO: INBIOCRI02242094, INBIOCRI02242096]. Est. Pitilla, 9 km S Santa Cecilia, P.N. Guanacaste, 700 m, 1059'28'N, 85²5'39”W, iv.1994, P. Rios, \#2844 [2§^, INBIO: INBIOCRI01793740, INBIOCRI01793753]. 
Puntarenas: Las Cruces Biological Station, Wales Trail, $1145 \mathrm{~m}, 8^{\circ} 47^{\prime} 21.5^{\prime \prime} \mathrm{N}, 82^{\circ} 57^{\prime} 43.1^{\prime \prime} \mathrm{W}$, 24-27.ix.2014, C. Weirauch, S. Leon, A. Knyshov, malaise trap, CR14 L3 MT2 [1 , UCRC: UCRCENT00491415; D4202]. Rancho Quemado, Peninsula de Osa, 200 m, 840'39"N, 83 33'53"W, 12.iii-3.iv.1994, A. Marin, \#2796 [1, INBIO: INBIOCRI01759946].

Related Material. Costa Rica: Heredia: $3 \mathrm{~km} S$ Pto. Viejo, $50 \mathrm{~m}, 10^{\circ} 26^{\prime} 00^{\prime \prime} \mathrm{N}, 84^{\circ}$

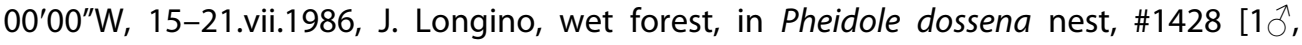
UCRC: UCRCENT00312201]. USA: FL: Miami-Dade, Miami, $1 \mathrm{~m}, 25^{\circ} 45^{\prime} 42^{\prime \prime} \mathrm{N}, 80^{\circ} 11^{\prime} 30^{\prime \prime} \mathrm{W}$, 29.v.1961, Stegmaier [1 $\widehat{\jmath}$, TAMU: UCRCENT00184060].

\section{Disclosure statement}

No potential conflict of interest was reported by the authors.

\section{Funding}

This study was supported by NSF grants DEB-1257733 and 1555808, and UCR Hatch grants to JMH.

\section{References}

Baker A, Heraty JM, Mottern J, Zhang J, Hines HM, Lemmon AR, Lemmon EM. 2020. Inverse dispersal patterns in a group of ant parasitoids (Hymenoptera: Eucharitidae: Oraseminae) and their ant hosts. Syst Entomol. 45:1-19. doi:10.1111/syen.12371.

Burks RA, Heraty JM, Dominguez C, Mottern JL. 2018. Complex diversity in a mainly tropical group of ant parasitoids: revision of the Orasema stramineipes species group (Hymenoptera: Chalcidoidea: Eucharitidae). Zootaxa. 4401:1-107. doi:10.11646/zootaxa.4401.1.1.

Burks RA, Mottern J, Heraty JM. 2015. Revision of the Orasema festiva species group (Hymenoptera: Chalcidoidea: Eucharitidae). Zootaxa. 3972(4):521-534. doi:10.11646/zootaxa.3972.4.4.

Darling DC. 1988. Comparative morphology of the labrum in Hymenoptera: the digitate labrum of Perilampidae and Eucharitidae (Chalcidoidea). Can J Zool. 66(12):2811-2835. doi:10.1139/z88409.

Harris RA. 1979. A glossary of surface sculpturing. Occ Papers Cali Dep Food Agr. 28:31.

Heraty JM. 1994. Classification and evolution of the Oraseminae in the Old World, with revisions of two closely related genera of Eucharitinae (Hymenoptera: Eucharitidae). Life Sci Contrib. 157:1-174.

Heraty JM. 2000. Phylogenetic relationships of Oraseminae (Hymenoptera: Eucharitidae). Ann Entomol Soc Am. 93(3):374-390. doi:10.1603/0013-8746(2000)093[0374:PROOHE]2.0.CO;2.

Heraty JM. 2002. A revision of the genera of Eucharitidae (Hymenoptera: Chalcidoidea) of the World. Mem Am Entomol Inst. 68:1-359.

Heraty JM, Burks RA, Mbanyana N, van Noort S. 2018. Morphology and life history of an ant parasitoid, Psilocharis afra (Hymenoptera: Eucharitidae). Zootaxa. 4482(3):491-510. doi:10.11646/zootaxa.4482.3.3.

Heraty JM, Wojcik DP, Jouvenaz DP. 1993. Species of Orasema parasitic on the Solenopsis saevissima complex in South America (Hymenoptera: Eucharitidae, Formicidae). J Hymenoptera Res. 2:169-182.

Herreid JS, Heraty JM. 2017. Hitchhikers at the dinner table: a revisionary study of a group of ant parasitoids (Hymenoptera: Eucharitidae) specializing in the use of extrafloral nectaries for host access. Syst Entomol. 42(1):204-229. doi:10.1111/syen.12206.

Murray E, Carmichael AE, Heraty JM. 2013. Ancient host shifts followed by host conservatism in a group of ant parasitoids. Proceedings of the Royal Society B: Biological Sciences. 280 (1759):20130495. doi:10.1098/rspb.2013.0495. 